
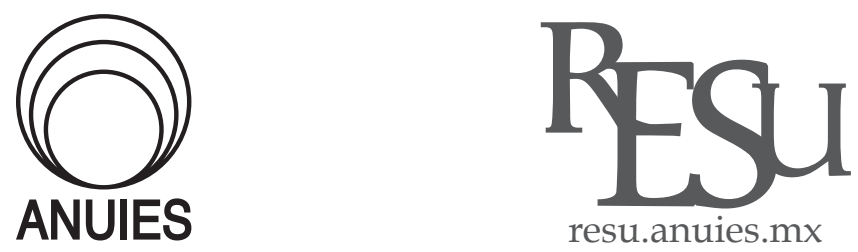

REVITA AEL
EDICACIÓN
SUPERIOR

ARTíCULO

\title{
La misión de la universidad en el siglo XXI: comprender su origen para proyectar su futuro
}

The role of the university in $21^{\text {st }}$-Century: understand its origin in order to project its future

\author{
Marta Ruiz-Corbella* y Ernesto López-Gómez** \\ * Universidad Nacional de Educación a Distancia. Madrid, España. \\ Correo electrónico: mruiz@edu.uned.es \\ Recibido el 29 de marzo del 2018; aceptado el 15 de enero del 2019.
}

\section{Resumen}

Este artículo es el resultado de la revisión de la literatura relevante sobre la idea de universidad. Defiende que el estudio sobre la génesis y la evolución de la institución universitaria aporta luz sobre su incierto futuro. A partir de un esquema argumentativo, reflexiona sobre su origen en la Edad Media, el sentido de la universidad como centro de investigación, para, finalmente, plantear tensiones y desafíos que enfrenta la universidad contemporánea. Pero esta institución debe armonizar las nuevas demandas que se le están exigiendo, sin renunciar a la identidad a la que se debe, si quiere seguir siendo universitas.

Palabras clave: Universidad, Educación Superior, Misión de la universidad, Relación educación superior-sociedad; Función de la universidad. 


\begin{abstract}
This article is the result of the review of relevant literature on the idea of university. Argues that the study of the genesis and evolution of the university can shed light on the uncertain future. Based on an argumentative scheme, it reflects on its origin in the Middle Ages, the sense of the university as a place for research, to finally raise tensions and challenges facing the contemporary university. This contemporary university is expected to harmonize the new demands with the necessary identity to which it is due, if it wants to remain universitas.
\end{abstract}

Keywords: University, Higher Education, Higher Education's mission; Higher Education's relationship with society, Higher Education's role.

\title{
Introducción
}

L a idea de universidad es una realidad compleja y difícil de abordar en los parámetros de un artículo. Desde luego, mucho y bien se ha pensado y escrito sobre ella, con distintos propósitos y finalidades, yuxtapuestos en ocasiones pero también integradores de una realidad que en la actualidad asume plurales escenarios y perspectivas. Es un tema recurrente en la producción científica, que en estos momentos ha vuelto a retomar de forma significativa su interés, más si cabe cuando se la está exigiendo comportamientos extraordinariamente competitivos (Ruiz-Corbella, 2006, Michavila, 2011; Pusser, Kempner, Marginson y Ordorika, 2012; Scott, Gallacher y Parry, 2016). En efecto, hoy universidad no se entiende sólo como un lugar o establecimiento físico, se asume que es mucho más que eso. Además, al romperse las coordenadas espaciotemporales surge un nuevo modelo, la universidad virtual, que está abriendo un nuevo modo de proceder y de ser de esta institución. Desde luego, la universidad es un centro de investigación y de enseñanza, una oportunidad formativa, una comunidad; permite comenzar el aprendizaje de una profesión de educación superior. No cabe duda de que las posibles interpretaciones, algunas más cercanas entre sí y otras un tanto distantes, son la consecuencia de la evolución de un concepto en el tiempo, de la historia de la idea de universidad, producto de las altas esperanzas que las personas y las sociedades ponen sobre ella. ${ }^{1}$

\footnotetext{
${ }^{1}$ Jacques Derrida. La universidad sin condición. Madrid: Trotta, 2002. "He aquí lo que podríamos, por apelar a ella, llamar la universidad sin condición: el derecho primordial a
} 
Sin una finalidad histórica prioritaria, se pretende comprender la génesis y la evolución de la institución universitaria con el objeto de proponer la misión de esta institución en este siglo xxI. Pero esta misión no puede desgajarse de lo que inicialmente fue, para y por qué fue creada, y la evolución que ha vivido a lo largo de la historia. Tampoco puede permanecer ajena a la realidad en la que está inserta y a la que debe responder, por lo que está obligada a evolucionar al ritmo de ésta, sin perder su propia identidad. Hace escasos años, la rectora de la Universidad de Harvard defendía que esta institución nos sigue dando la oportunidad de ver más allá del aquí y ahora, ya que la esencia de la universidad es su capacidad de unir pasado y futuro.

Universities must help define aspirations and possibilities for the long term. Even as they engage the present, they must help us transcend the immediate and the instrumental to explore where human civilization has been and where it hopes to go (Faust, 2013).

Pero reparemos en esta evolución desde su sentido y misión inicial.

\section{Primero, la universidad como comunidad}

La palabra "universidad", del latín "universîtas, -ãtis", es la síntesis de unus (uno) y verto (girado), a saber: "girado hacia uno". Un breve recorrido etimológico constata que "verto" refiere a "verter", que es derramar y arrojar, y también a "versar" que es dar la vuelta a algo, tratar un asunto; alguien versado, es alguien ilustrado. De verso (verto) procede universo, que es el todo, la totalidad y lo que a ello rodea (unus, versus). Y así llegamos a universidad, como totalidad de los estudios y de los estudiosos "magistrorum et scholarium" que a ello se empeñan, compartiendo raíz con universo y universal.

Así, desde una aproximación etimológica, universidad sería el punto donde todo se une y gira; es totalidad de estudios y estudiosos - profesores y estudiantes - que se mueven, que cambian, pero, a la vez, mantienen la unidad. Así, unión y unidad reclaman la idea de comunidad (communitas), lo que nos lleva a comprender que universidad vendría a ser búsqueda constante en común-unidad. No en vano, universitas se vino a identificar con una corporación que se orientaba hacia una meta común, en la que sus miembros "han de tener intereses en común y un estatuto legal independiente" (Cob-

decirlo todo, aunque sea como ficción y experimentación del saber, y el derecho a decirlo públicamente, a publicarlo." p. 14. 
ban, 1975, p.23), que es exactamente lo que garantiza su identidad: universitas magistrorum et scholarium "comunidad de maestros y escolares".

A la hora de comprender el origen de la universidad, el historiador medievalista Le Goff indica que precisamente “[...] el siglo XIII es el siglo de las universidades, porque es el siglo de las corporaciones" (Le Goff, 1993, p. 71). Ahora bien, que fuera a comienzos del siglo XIII cuando floreció propiamente la institución universitaria, no quiere decir que su actividad genuina, el cultivo de los saberes y la búsqueda de respuestas en torno a la verdad, belleza y bondad, no se hiciera ya años atrás (Bayen, 1978).

La prehistoria de la universidad cabe estudiarla desde aproximaciones valiosas en un contexto lejano al europeo, como la Casa de la Vida en Egipto o las Escuelas Islámicas de Sabiduría (Ebied y Yung, 1975). Ahora bien, consideramos que no puede afirmarse que la universidad se origine en Egipto, sencillamente porque éstos eran únicamente establecimientos de enseñanza. De igual forma, cabe tener en cuenta la fecunda tradición clásica cultivada en sus más sobresalientes instituciones: La Academia, El Liceo o El Jardín griegos, o la influyente Educación Superior romana. En suma, debemos reconocer que nuestra universidad, en su origen medieval, es heredera de la tradición clásica, de aquellas escuelas de pensamiento y filosofía - amor a la sabiduría - consolidadas por grandes maestros, que fueron capaces de crear un espacio para la discusión sobre temas relevantes y dejar un incalculable legado intelectual con notable influencia en el saber contemporáneo. Así lo reconoce Werner Jaeger (2007) al referirse a la importante herencia clásica, especialmente griega, para el desarrollo cultural de Occidente, donde quizá sea la universidad la cúspide de tal florecimiento y el principal baluarte que ha consolidado buena parte de la identidad cultural europea.

En este sentido, el origen de la universidad presenta diversas causas muy ligadas al contexto medieval. La primera de ellas hace referencia a aspectos sociales y científicos, fruto del abundante sustrato cultural de la época, cada vez mayor, debido a las traducciones, a los comentarios de textos clásicos y a la presencia de grandes maestros. En segundo lugar, la pluralidad de diversos mundos culturales (griego, latino, cristiano, judío y árabe) tiene una gran incidencia, ya que crearon un espacio propicio para el cultivo de los saberes. Finalmente, se detectan causas laborales y académicas, en el sentido corporativo del contexto medieval, donde surge la necesidad de formar una corporación y una comunidad de maestros y escolares, como expresión del sentido gremial de la sociedad del momento y como institución que enfatiza lo común (Carañana, 2012). Obedece a una combinación de poderosas tendencias sociales: "[...] la reactivación del mercantilismo, el crecimiento de las ciudades y la clase media urbana, la burocratización y el renacimiento inte- 
lectual del siglo xII" (Scott, 2006, p. 9). Se llega a definir como "la institución europea par excellence" (Rüegg, 2004). Tal es así, que Julio Ruiz Berrio (1986) matizaba que por European universities se entendían tanto las instituciones alojadas en el territorio de Europa, como las que están ubicadas en cualquier punto geográfico pero que mantienen, en su misión y en su organización, ese carácter europeo. Claramente, la universidad nació como comunidad de profesores y estudiantes a los que se han concedido ciertos derechos tales como “[...] la autonomía administrativa, la elección y la realización de los currícula (materias de estudio), los objetivos de investigación así como la recompensa de los títulos reconocidos públicamente" (Rüegg, 1994, p. 19).

Será a partir del Renacimiento cuando triunfe la voz universidad para designar a los centros de cultura superior (Negrín y Vergara, 2014). En estas instituciones de cultura superior los saberes se orientaron a una formación general, esencialmente humanística, y a la formación de profesionales en una sociedad teocéntrica (Villa, 2005; Esteban y Martínez, 2012). No en vano se ha dicho en numerosas ocasiones que la universidad es hija de la cristiandad europea medieval cuyo propósito era la búsqueda de la verdad. Pero los cambios sociales, económicos, políticos [...] crearon un nuevo escenario de enorme complejidad entre la iglesia romana y los gobiernos seculares que demandaban tanto sacerdotes educados como abogados, médicos y empleados de negocios altamente preparados. Esta demanda abrió las puertas de la universidad a la enseñanza y a las profesiones liberales. En efecto, en su origen fue un enclave para la transmisión de los saberes, una raíz universitaria medieval cargada de un fuerte compromiso docente, en definitiva, con la enseñanza (Scott, 2006). A la vez formaba a los profesionales que requería esa sociedad, por lo que su finalidad tenía un inmediato sentido de bien común.

\section{Después, la universidad como morada de la investigación}

Durante el siglo Xvi hubo cierta continuidad con el modelo medieval, si bien es cierto que comienzan a apreciarse diferencias que, por las pretensiones de este trabajo, no abordaremos. A finales del XVII y principios del XVIII, comienza a ser cada vez más notable la ruptura entre filosofía y ciencia, fuentes de sentido de la universidad del momento. En efecto, hasta entonces existía una concepción unificada del saber donde la búsqueda de lo verdadero (verum), de lo bueno (bonum) y de lo bello (pulchrum) estaban relacionadas, cuando no eran idénticas. 
El nuevo movimiento defendía que el único camino al conocimiento de la verdad era la teoría que basaba su método en la inducción a partir de observaciones empíricas que pudieran ser repetidas y verificadas en distintos momentos y diversos contextos. El nuevo científico insistía en que su trabajo tenía que ver con la búsqueda de lo verdadero desde dicho método, dejando para los filósofos la tarea de la búsqueda de lo bueno y lo bello, lo que conllevó la ruptura cada vez más determinante entre ambos (Wallerstein, 2005). Esto es lo que Charles P. Snow (1987), años después, vendría a llamar "las dos culturas", los científicos por un lado y los intelectuales por otro, con altas dosis de incomprensión y falta de entendimiento mutuo, que aún se mantiene.

Pese a este intenso debate, las universidades continuaron con el modelo medieval hasta iniciado el XIX, momento en el que se comenzó a pensar que la universidad era improductiva en relación a los nuevos modos de producción económica, consideración fruto del advenimiento de la sociedad industrial (García Garrido, 2009). Como señala Daniel Fallon (1980), durante el siglo XVIII las universidades se describen como medievales, "atrophied in a tradeguild mentality", donde el conocimiento estaba fijado en un sistema cerrado. En este contexto se fue llevando a cabo, de forma paulatina, una clara modernización originada por: la disección de los saberes y la emergente especialización científica, el acercamiento de la universidad docente y formativa medieval hacia una más investigadora y académica, la distancia creciente entre la universidad y la tradición cristiana que la vio nacer y, a su vez, la mayor cercanía de la universidad al Estado y, en cuarto lugar, el reconocimiento de los principios básicos académicos de libertad de pensamiento y libertad docente.

Ahora bien, y pese a la pluralidad de medidas que emergen en esos años en torno a la universidad, hay una que predomina sobre el resto: el modelo alemán, liderado por Humboldt e institucionalizado en la Universidad de Berlín en 1810 bajo el rectorado de Fichte (Anderson, 2000). Esta "enlightenment university" (Fallon, 1980) se configura desde las bases conceptuales de la "universidad alemana": el conocimiento por sí mismo (Humboldt), el renacimiento de la nación alemana a través de la educación (Fichte), la relación entre el Estado y la universidad y la noción de libertad académica (Schleiermacher), así como el papel de la filosofía y de los héroes históricos en la historia de las naciones (Schelling), fundamentos de la nueva universidad (Kwiek, 2006). Todos ellos representan las claves de lo que se ha dado en llamar el modelo humboldtiano o centroeuropeo.

Mitchell G. Ash (2006) identifica cuatro elementos fundamentales a destacar en este modelo de universidad: en primer lugar, la libertad de enseñanza $y$ de aprendizaje (Lehr-und Lernfreiheit), que refiere, por una parte, al derecho 
de los estudiantes para elegir a los profesores y, por otra, a la capacidad de éstos para decidir qué y cómo enseñar. Esto trajo consigo una ruptura radical y constituye la génesis de lo que se ha conocido como libertad de cátedra.

En segundo lugar, la unidad de enseñanza e investigación (Einheit von Lehre und Forschung), en la que investigar, enseñar y aprender son procesos que requieren un alto grado de colaboración, y se necesitan mutuamente. Sin duda, resultará vital para la actividad científica que "[...] en un solo espacio se reúnan las tareas docentes e investigadoras. Esto implicará una mejora sustantiva en ambas, pues las clases mejorarán al ser impartidas por docentes que se encuentren al mismo tiempo inmersos en el desarrollo de la ciencia, y la investigación se verá favorecida por el hecho de ser discutida en las clases" (Villa, 2005, p. 279). Decía Humboldt que el profesor de universidad no es un maestro ni el estudiante un educando, sino alguien que investiga por sí mismo, guiado y orientado por el profesor. Esta relación permitía que los estudiantes estuvieran en condiciones de desempeñar por sí mismos una labor científica (Hohendorf, 1993), por lo que esta idea de universidad reclama un compromiso autoformativo por parte del conjunto de estudiantes, quienes mediante el contacto y la supervisión de los profesores - expertos disciplinarios - debían desarrollar una tarea investigadora. Con Humboldt lo más valioso es que sea uno mismo quien consiga algo o lo desarrolle. Más allá de la utilidad se valora el desarrollo del "yo", al reconocer la formación de la individualidad como meta última del universo (Piedras Monroy, 2008). De ahí la máxima humboldtiana: "[...] soledad y libertad para los que aprenden y para los que enseñan" (Bonvecchio, 2002, p. 78). En efecto, Humboldt defenderá que antes que la asistencia a las clases magistrales, es más relevante la reflexión científica en soledad y libertad (Piedras Monroy, 2008).

En tercer lugar está unidad de la ciencia (Einheit der Wissenschaft), fruto de un nuevo enfoque del trabajo intelectual, abierto al aprendizaje, capaz de pensar más allá del enfoque de enseñanza sobre lo descubierto. No se trata entonces de una cosa encontrada, sino de algo inacabado y buscado, de que la universidad afronte "[...] el tratamiento de la ciencia como un problema aún no resuelto del todo que debe ser objeto de constante investigación" (Hohendorf, 1993, p. 714). Aparece así, como baluarte de este modelo, el concepto de investigación, que tiene que ver con el compromiso por indagar y avanzar - no sólo transmitir - en los saberes, de tal forma que la universidad esté en continua actualización y movimiento. Como nos dirá Daniel Fallon (1980, p. 19), en la universidad han de estar "[...] los mejores intelectos disponibles que tengan la libertad de llevar a cabo sus investigaciones hasta las últimas consecuencias". Así, cada catedrático de la Universidad de Berlín dirigió un instituto o seminario de especialización curricular con una cuidada pirámide 
de profesores jóvenes y estudiantes. Es en este escenario donde aparece el profesor especializado, que cultiva un determinado ámbito científico, que produce conocimientos desde una investigación rigurosa sobre una parcela concreta del saber (Piedras Monroy, 2008). Esta erudición cultivada por el profesor alemán fue reconocida y valorada internacionalmente, con una mayor atención en el campo de la historia, la filología y la química. Su influencia es debida, en parte, a la importancia de visitar universidades extranjeras y de explorar contextos científicos lejanos al propio, pues, como diría Humboldt, para las ciencias no existen las fronteras (Villa, 2005).

En suma, se diseñó una universidad en la que la formación se logra a través de la ciencia (Bildung durch Wissenschaft), que se entiende como un proceso firme e inclausurable que requiere el concurso de todas las fuerzas creadoras que toman parte en ella y que implica una incansable búsqueda común. A la vez, el trabajo académico tenía grandes pretensiones éticas, pues a través de él se trataba de ennoblecer a las personas y de encaminarlas a un servicio abnegado para el bienestar de la comunidad (Hohendorf, 1993). Ahora bien, Humboldt era consciente de que la universidad debía proveer una cultura general, sustentada en el saber científico, antes que una formación profesional especializada (Morín, 2010).

\section{Un paso más, la universidad más allá de la investigación. Hacia un modelo integrador}

Al adentrarnos en el s. XIX, encontramos perspectivas que, desde diversos enfoques, hicieron énfasis en otras finalidades para la educación universitaria. En primer lugar, la importancia concedida ya por entonces a la formación de profesionales altamente especializados, que asume en sentido radical el modelo napoleónico, antítesis de lo que hasta entonces se entendía por universidad. Se trataba de formar profesionales con una clara vocación de servicio público donde la diversidad y la heterogeneidad era la nota dominante. La universidad napoleónica representó un organismo nacional centralizado e independiente que relegaba la educación literaria y científica fundamental - liberal education - a las escuelas secundarias y que entendía que enseñanza e investigación debían estar delimitadas y separadas. La educación universitaria se configura de este modo como un instrumento de poder y estabilidad política. Así lo indica José Luis García Garrido (2009, p. 13) al referir que "[...] la Université de France no es sólo una institución universitaria, sino todo un sistema educativo encargado de la educación pública en todo el Imperio. Se trataba de conservar y afianzar del mejor 
modo posible el nuevo orden social impuesto, tras la Revolución, por las nuevas autoridades".

En segundo lugar está el modelo británico cuya génesis refiere al estilo universitario de Oxford y Cambridge. Esta visión de la universidad propone un horizonte formativo basado en el desarrollo personal de los estudiantes, con un especial énfasis en la formación de las élites, como futuras responsables del gobierno de lo común y del porvenir político, cultural y económico de la sociedad. Se entiende que la formación intelectual es el primer objetivo de la actividad universitaria, valorando la aspiración del individuo al saber y entendiendo a la universidad como medio de formación. En suma, es la "excelencia intelectual" (Newman, 1996) como cualidad distinguible de la universidad. La formación universitaria vendría a ser el conocimiento unido e integrado de las disciplinas, de modo que se entiende el carácter indispensable y necesario y, a su vez, la limitación de cada una de las áreas del conocimiento para la comprensión de las cosas. La unidad del conocimiento, o si se quiere, la unidad del entendimiento, implica que cada una de las disciplinas académicas permite avanzar en el conocimiento de algún aspecto particular, pero la auténtica comprensión implica ir más allá de las diferentes disciplinas - historia, literatura, matemáticas - y estudiar cómo cada una de ellas se relaciona con el resto. El propio Newman destacará la labor de la teología como ordenadora del conocimiento y el entendimiento, lo que ofrece alguna pista sobre su respeto al modelo medieval (García Garrido, 2009).

En relación a la investigación, Newman argumentará en sus conocidos discursos que es una tarea de instituciones distintas a la universidad, postura contraria al modelo alemán entonces emergente y al norteamericano posterior. Vendría a decir que las cualidades propias de la mente de los investigadores especialistas son incompatibles con los de una mente educada, meta de la universidad formativa que propone. Y respecto a la formación profesional, el modelo británico es distante de los propósitos de la universidad napoleónica, ya que la formación intelectual viene a suplantar a la profesional, de no ser así la universidad se desnaturalizaría.

No cabe duda de que la universidad moderna se debatió entre ser un espacio para la investigación - humboldtiano-, la formación de profesionales - napoleónico - y la educación intelectual propia de una universidad formativa - británico - Y Y con la perspectiva que el tiempo aporta, se optó por ser la morada propia de la investigación (Wittrock, 1991). Esta visión de la universidad fue importada a otras regiones, especialmente la norteamericana, donde los comienzos de algunas de sus universidades como John Hopkins y Cornell University contaban con personal académico que había sido formado en universidades alemanas (Fallon, 1980). Así, en la segunda mitad del XIX, el 
modelo humboldtiano influye en universidades americanas $y$, aunque no fue sencillo incorporar la función investigadora durante décadas, será tras la Segunda Guerra Mundial cuando las mejores universidades norteamericanas se centraron en ese enfoque investigador.

A la vez, a partir de mediados del siglo xx, la universidad tradicional de élites se convierte en una universidad de masas (García Garrido, 2009). Este proceso ha evidenciado que el acceso a la universidad de élite - a privilege of birth or talent or both-, a la universidad de masas - a right for those with certain qualifications - y la universidad universal - an obligation for the middle and upperclasses - ha sido muy variado, estrechamente relacionado con la universalización de la educación secundaria y post-secundaria (Trow, 2006). Es aquí donde tiene sentido traer a colación la génesis del modelo norteamericano que desde sus comienzos ofreció una mayor apertura a las demandas sociales. Además impulsa el vehículo principal del conocimiento y el desarrollo de la ciencia: la lengua inglesa, identificada por Philip G. Altbach (2007) como auténtico imperio. De hecho, Walter Rüegg (2004, p. 13) indica que "[...] the higher education systems of continental Europe have never been able to combine the general education of undergraduate students with scientific teaching humboldtian, as they do in the best anglo-american universities". Se trata de un modelo, el norteamericano, que ha sabido asumir varias funciones: (1) producir avanzados conocimientos científicos especializados y formar a los jóvenes para ser buenos investigadores; (2) proporcionar una educación general para la "ciudadanía educada"; (3) generar una visión y un conocimiento de los problemas humanos y sociales así como desarrollar en los estudiantes la capacidad de juicio crítico; (4) preparar a los estudiantes para prácticas profesionales y académicas con gran especialización (Parsons y Platt, 1973).

Las dos primeras funciones tienen que ver con la naturaleza propia de las universidades - investigación y enseñanza - y se observa en ellas una clara influencia alemana, especialmente en la primera, si bien Frank H. T. Rhodes (2001) reconoce que las universidades de investigación representan un número relativamente pequeño ("quizás 125 o menos") de entre todas las universidades de los Estados Unidos. Este autor realiza una importante defensa de las universidades de investigación y el papel que desempeñan, y propone que la investigación universitaria debe apuntar más alto, tanto para cumplir con su antigua misión como para dar forma a la visión de futuro de la sociedad americana.

En la segunda función, se aprecia la tradición británica, a partir de la idea de educación liberal. Así, Alfred N. Whitehead (1951) indicará que la educación universitaria tiene en el cultivo de la inteligencia - cultivation of mental power - su gran referente. Para este autor, el espíritu de generalización debe 
dominar la universidad y es "[...] en el cultivo de la inteligencia donde coinciden el interés teórico y la utilidad práctica" (Whitehead, 1951, p. 38).

La tercera y la cuarta funciones vendrían a ser requisitos y encargos que la sociedad espera de estas instituciones desde un punto de vista ético y comunitario, económico o de desarrollo productivo. En este sentido, Martha Nussbaum (1997) sugiere que las universidades deben educar a sus ciudadanos con el objetivo de favorecer una democracia plural a partir de dos grandes propósitos. El primero de ellos tiene que ver con que los estudiantes sean capaces de valorar críticamente sus propias convicciones, actitudes y costumbres a través del razonamiento lógico, la argumentación y la reflexión, y el segundo, al asumir una actitud cosmopolita en la aldea global que permita desarrollar actitudes valiosas de empatía e imaginación, donde la formación liberal se centre en "[...] la tarea de activar en cada estudiante una mente independiente y producir una comunidad que pueda verdaderamente razonar en conjunto sobre un problema y no simplemente intercambiar alegatos" (Nussbaum, 1997, p. 40).

El modelo de universidad norteamericana ha venido a ser un variado crisol, denominado en ocasiones como multiversidad, 'multiversity or pluralistic university'. Este modelo referencial se caracteriza, al menos en teoría, por armonizar investigación, enseñanza, educación liberal y formación de profesionales, con una amplia apertura a la sociedad y al contexto del momento, donde "[...] habitan diversas comunidades, con intereses variados y a veces incluso conflictivos" (Kerr, 1992, p. 18). Sin duda, estas funciones han creado tensiones y presiones en torno al quehacer universitario $\mathrm{y}$, nos preguntamos si acaso: “ ¿es universidad aquella institución que se dedica casi exclusivamente a la formación profesional y se desvincula de la formación humanística o viceversa, o que sólo se dedica a la docencia y no a la investigación?" (Esteban y Martínez, 2012, p. 78). ¿Tiene sentido una universidad para todos? ¿No genera el acceso libre o masificado frustraciones personales y profesionales de hondo impacto social? ¿No era la misión de la universidad una formación de élites?

\section{La universidad de un futuro ya presente. Continuidad, cambio y transformación}

Durante el siglo xx las diferentes ideas sobre la universidad continúan manteniéndose y dirigiendo la política educativa universitaria expandiéndose por los diferentes países. Pero será a mediados de siglo cuando irrumpan dos factores que condicionan radicalmente el desarrollo de esta institución: 
el derecho a la educación superior de toda la población, que conlleva la masificación universitaria, y el reconocimiento de la educación a lo largo de la vida, que abrió a la institución universitaria a otros escenarios, otros tiempos y contenidos de formación. Se acudió a las universidades para responder a los problemas de la sociedad cada vez más diversos y más rápidamente cambiantes, que exigen mayor especialización. Se entra en una época muy compleja, en la que accedían a la universidad un número cada vez más creciente de estudiantes, pero en la que todavía no se contaba con suficientes recursos, con un sólido nexo con la sociedad, y en la que las funciones de investigación y formación avanzaban de forma paralela y a diferente ritmo.

Fueron años en los que la prioridad se centró en solucionar los problemas más inmediatos, sin proyectar un futuro sólido a esta institución. Esto generó la necesidad de plantear un nuevo modelo de universidad, razón de que se esté revisando a nivel mundial el sistema universitario. En estas reformas coinciden todas las instancias supranacionales, especialmente a partir de 1990, en la que se promueve una institución universitaria capaz de responder tanto a las exigencias del ámbito económico como a las necesidades sociales. Era necesaria una nueva educación superior, capaz del desarrollo social y económico a través de la formación de los profesionales en competencias, la transferencia tecnológica y el desarrollo permanente de los conocimientos. Poco a poco, a través de diferentes informes y documentos institucionales, se mostró que la universidad no podía quedarse al margen de la sociedad del nuevo milenio, en la que se preveía “[...] una demanda de educación sin precedentes, acompañada de una gran diversificación de la misma, y una mayor toma de conciencia de la importancia fundamental que este tipo de educación reviste para la construcción del futuro" (UNESCO, 1998, p. 1).

El inicio del nuevo siglo estuvo marcado por enormes transformaciones sociales, económicas, demográficas, políticas, tecnológicas, que han influido, y continúan haciéndolo, en el modo de trabajar, de relacionarse, de entender la docencia y la investigación. Este escenario ha contribuido también en un nuevo paso hacia el cambio radical de la universidad. Ahora, a pesar de esta realidad imparable, que sabemos que debemos abordar, seguimos pensando en una universidad "tradicional". Estamos viviendo en un híbrido, al continuar actuando con los esquemas de esa institución tradicional incorporando algunas exigencias que demanda el presente. Se trata de dos discursos que parecen no encontrarse. Sin duda, toda universidad debe tener impacto en el mundo que habitamos hoy, pero su responsabilidad se extiende mucho más allá. Debe colaborar a definir aspiraciones y posibilidades a largo plazo, sin perder el presente; debe ayudarnos a trascender lo inmediato y lo instrumen- 
tal para explorar dónde ha estado la civilización humana y hacia dónde debe ir; debe preparar para hacer posible nuestro futuro.

Sin embargo, aún no se ha sabido centrar el debate en lo importante: qué tipo de educación superior es la que se quiere y cuál es la que está implantándose. Es decir, reflexionar sobre los objetivos que persigue, la formación que oferta, los logros que alcanza, para valorar su pertinencia en la actualidad. Sin duda, la fusión, por un lado, del paradigma del aprendizaje a lo largo de la vida y la democratización de su acceso, y, por otro, la globalización y la revolución tecnológica, ha propiciado nuevos modelos de educación superior al romper las barreras temporales y espaciales de estas instituciones, convergiendo todas ellas en el espacio de la virtualidad; diversificar los proveedores de educación superior (públicas, privadas, transnacionales, corporativas, etc.); reconocer la dimensión formativa de cualquier escenario e incorporar nuevos modelos de organización y planificación curricular.

De este modo el escenario de aprendizaje se va transformando de forma vertiginosa, lo que conlleva la revisión de los diseños formativos implantados. Deben valorarse si éstos responden a la formación que se exige a la persona, al ciudadano y al profesional de este milenio para saber afrontar un futuro marcado por la complejidad y la incertidumbre ante el cambio cada vez más acelerado. Estamos ante la transformación de una institución de transmisora del saber, a gestora del conocimiento (Ruiz-Corbella, 2016). Ahora bien, no olvidemos tampoco que todo este proceso de cambio no hubiera sido posible sin la irrupción de las tecnologías de la información y la comunicación; sin ellas no podríamos diseñar estos escenarios emergentes de aprendizaje que facilitan nuevos recursos en los que nos apoyamos, diseños de formación más flexibles y personalizados, diversificación de los escenarios donde "ocurre" el aprendizaje.

Pero lo relevante es que esta nueva transformación está basada en el conocimiento, en la información, las comunicaciones y la innovación tecnológica, que afecta a todos los ámbitos de la vida de las personas, lo que va a exigir un cambio radical de la economía. Esto originó que la educación se centre en la competencia digital, en la productividad económica (desarrollo social, investigación e innovación) y la creación de empleo (empleabilidad, emprendimiento). Se trata del conocido "triángulo del conocimiento" (knowledge triangle) que vincula la educación, la investigación y la innovación, empujando a la universidad a la lógica de la economía (Maassen y Stensaker, 2011). De ahí que se inste a las instituciones de educación superior que se forme en competencias, o que entre los indicadores de la calidad de estas universidades prime lo relativo a la empleabilidad de sus titulados. O su capacidad de emprendimiento, 
que se concreta como innovación tecnológica y su capacidad de comercialización. En suma, se abre una nueva forma de entender la universidad.

\section{Conclusiones}

Llegados a este punto cabe referir una idea que, desde luego, no es nueva: el germinar de la universidad, en la Edad Media, tiene su sentido junto a la idea de comunidad de maestros y escolares con voluntad de entender y aprender los saberes. En efecto, desde su origen ha querido consolidarse como eje de desarrollo social, consciente de su papel comunitario, cultivando un eje horizontal (saber más cosas, ampliar el horizonte de conocimientos) y vertical (saber más sobre una de ellas) que, por otro lado, han enfrentado dos posiciones difícilmente reconciliables: la educación liberal y la especialización. Este enfrentamiento se ha resuelto con el valor predominante hacia el modelo alemán que ha prevalecido a lo largo del xIx e inicios del xx (Wittrock, 1991), debido a "[...] su apuesta por la investigación científica como pivote de la vida universitaria, lo que sin duda ha resultado decisivo para el progreso de la ciencia y de la tecnología; y [...] su atractivo ante profesores y alumnos" (García Garrido, 2009, p. 13).

No obstante, una parte de la literatura ha mostrado una posición crítica respecto al modelo alemán, que si pierde la capacidad de pensar acerca de los fines, deteriora la de autocrítica (MacIntyre, 2009). Además, se ha reprochado fuertemente la distancia que en este modelo se justifica entre la sociedad y la universidad, valorándolo como elitista, con excesiva uniformidad y escasa diversidad de servicios. De hecho, en el conocido Informe Tunning leemos que "[...] el enfoque humboldtiano ha sido sustituido por un enfoque más centrado en la sociedad" (González y Wagenaar, 2003, p. 248). Al final, nos dirá Borja Villa (2005), la universidad humboldtiana es correcta en la teoría pero no vale para la práctica, debido a la gran distancia de sus ideales con el interés que la sociedad tenga respecto a ellos. En efecto, la demanda social ha provocado que el ideal humboldtiano pierda actualidad y vigencia, desde una lógica de masificación en la enseñanza superior (Trow, 2006), la irrupción de las tecnologías y las grandes trasformaciones económicas. Esto ha supuesto, a su vez, que la universidad deje de ser una torre de marfil y se desarrolle de forma más pragmática.

Derek Bok (1982), a su vez, sugiere enfoques que permitan a la institución académica servir a la sociedad y continuar con su misión principal de enseñanza e investigación. De hecho, autores como Frank H. T. Rhodes (2001), han propuesto que el destino de la universidad como proveedora de conoci- 
mientos es la fuente de sentido para las universidades de investigación. Estamos ante una visión necesaria, si se quiere lograr una universidad excelente (Readings, 1999), que pasa por recuperar el pensamiento sin estar sujetos a criterios económicos y de mercado (Harris, 2008).

Está claro que la misión de la universidad debe conjugar la docencia y la transmisión de la cultura, junto con la investigación y la transferencia del conocimiento y la dimensión social de esta institución. A lo largo de las últimas décadas se ha dado más relevancia a la investigación, a la transferencia del conocimiento, a la innovación dirigida al desarrollo económico de las naciones, que a la docencia, o a la dimensión social, o a la transmisión de la cultura. No es cuestión de ver si damos prioridad a una u otra misión, sino de desarrollar todas ellas de forma equilibrada y convergente (López-Gómez, 2015): "Sin la relación profesor-alumno, la Universidad deja de serlo, pero sin la creación de nuevos conocimientos a través de la investigación, no es Universidad" (Pétriz y Rubiralta, 2017, p. 47).

Y volvemos al inicio, recuperar la responsabilidad ineludible de toda universidad de liderar la generación de conocimientos, de destrezas, de valores, todos ellos necesarios para afrontar los problemas sociales, económicos, ambientales, etc., mejorando la capacidad de la ciudadanía para responder a estos desafíos (Paleari, Donina y Meoli, 2015). Como ha propuesto recientemente Ronald Barnett (2017), una ecological university que atienda al menos siete ecosistemas: knowledge, the economy, social institutions, learning, individual persons, culture, and the natural environment. En definitiva, cada uno como estudiante, profesor, investigador o gestor - que sea y construya universidad. Debemos orientarnos a un modelo pedagógico, de investigación y docencia, dirigido al bien común, sin ser ajenos al entorno en el que vivimos (Pusser, Kempner, Marginson y Ordorika, 2012; Marginson, 2016).

Estamos ante un claro camino para construir y comprender la universidad como comunidad ética, ya que desde este modelo formativo estamos apostando por una institución capaz de formar excelentes profesionales - buen nivel de formación, capacidad de innovar e internacionalización -, guiados a la vez por una ética de la responsabilidad orientada al logro de resultados, no sólo profesionales sino también sociales y éticos (Teichler, 2015; Zgaga, 2014). Mejorar la calidad y abordar la renovación en la universidad desde esta perspectiva debe integrar necesariamente objetivos relacionados con la excelencia en la docencia, los aprendizajes de los estudiantes, la gestión de la institución, la investigación y la transferencia del conocimiento, no en abstracto sino en concreto, tanto en la propia institución, igual en sus ámbitos de actuación que en las acciones que se desarrollan en todo escenario de la vida universitaria. 
En efecto, la evolución de la idea y la misión de la universidad desde sus orígenes hasta el momento actual es una realidad. Ahora, identificamos unas características que la definen que, de una forma u otra, siempre han estado presentes y deben permanecer para garantizar la continuidad de esta institución. Deberá cambiar de acuerdo a las necesidades y exigencias de cada momento, aceptando la plural idea de universidad, sin olvidar que:

[...] universities generate a wide diversity of outputs. In research, they create new possibilities; in teaching, they shape new people. The two interact powerfully to generate emergent capacities that are adapted to the needs of the times, embodying and creating the potential for progress through the ideas and the people that will both respond to and shape an as yet unknown future (Boulton y Lucas, 2008, p. 4).

Pero ante ese futuro incierto, " [...] la Universidad no puede permanecer ajena a la realidad en la que vive, sino que debe ser motor de desarrollo y de cohesión social" (Ruiz-Corbella y Bautista-Cerro, 2016, 185). Estamos ante instituciones que ya no tienen el monopolio del saber, ni de la ciencia, aunque sí la capacidad para liderar la investigación y el desarrollo del conocimiento junto a otros. Y es en este punto donde deben saber seguir siendo fieles a su razón de ser, a la tensión que las caracteriza: la búsqueda de la verdad, la formación, la universalidad y la colaboración (Esteban y Martínez, 2012).

\section{Referencias}

Altbach, Philip G. (2007). The Imperial Tongue: English as the Dominating Academic Language. Economic and Political Weekly, 8, 3608-3611.

Anderson, Robert. (2000). Before and after Humboldt: European universities between the eighteenth and the nineteenth century. History of Higher Education Annual, 20, 5-14.

Ash, Mitchell G. (2006). Bachelor of What, Master of Whom? The Humboldt Myth and Historical Transformations of Higher Education in German-Speaking Europe and the US. European Journal of Education, 41 (2), 245-267, doi 10.1111/j.1465-3435.2006.00258.x

Barnett, Ronald. (2017). The Ecological University: A Feasible Utopia. New York: Routledge.

Bayen, Maurice. (1978). Historia de las universidades. Barcelona: Oikos Tau.

Begley, Ronald. (2005). Medieval Education. New York: Fordham University Press.

Bok, Derek. (1982). Beyond the ivory tower: social responsability of the modern university. Cambridge: Harvard University Press. 
Boulton, Geoffrey y Lucas, Colin. (2008). What are universities for? [en línea]. [Consultado el 26/04/2017] Disponible en: http://www.leru.org/files/publications/What_are_universities_for_September_2008.pdf

Bonvecchio, Claudio. (2002). El mito de la universidad. México: Siglo xxi.

Carañana, Joan Pedro (2012). La teoría y la práctica en la universidad medieval. Cuadernos del Instituto Antonio de Nebrija, 15/2, 139-161

Cobban, Alan B. (1975). The Medieval Universities: Their Development and Organization. Londres: Methuen.

Ebied, Rifaat y Young, M. L. J. (1975). Did the Arabs invent the university? Times Higher Education Supplement, may.

Esteban, Francisco y Martínez, Miquel. (2012). ¿Son universidades todas las universidades? La universidad como comunidad ética. Bordón. Revista de Pedagogía, 64 (3), 77-92.

Fallon, Daniel. (1980). The German University. A heroic ideal in conflict with the modern world. Colorado: Associated University Press.

Faust Drew. (2013). To Seize an Impatient Future. [conferencia]. Sanders Theatre, Harvard University, Cambridge, Mass., September 21. [en línea]. [Consultado el 06/03/2017]. Disponible en: http://www.harvard.edu/president/ speech/2013/to-seize-impatient-future

García Garrido, José Luis. (2009). Futuro de la universidad o universidad del futuro. Revista Fuentes, 9, 9-25.

González, Julia y Wagenaar, Robert. (eds.). (2003). Tuning Educational Structures in Europe. Informe Final. Fase 1. Bilbao: Universidad de Deusto.

Harris, Suzy. (2008). La dimensión internacional de la universidad: entre el modelo económico y el cultural. Estudios Sobre Educación, 15, 87-98.

Hohendorf, Gerd. (1993). Wilhelm von Humboldt (1767-1835). Perspectivas: Revista Trimestral de Educación Comparada, 3-4, 707-719.

Jaeger, Werner. (2007 [1942]). Paideia: los ideales de la cultura griega. México: Fondo de Cultura Económica.

Kerr, Clark. (1992). The uses of university. Cambridge: Harvard University Press.

Kwiek, Marek. (2006). The university and the state. A study into global transformation. Frankfurt, New York: Peter Lang.

Le Goff, Jacques. (1993). Los intelectuales en la Edad Media. Madrid: Gedisa.

López-Gómez, Ernesto. (2015). Conectando investigación y docencia en la Universidad: teaching research nexus. Teoría de la Educación. Revista Interuniversitaria, 27 (2), 203-220, doi: http:/ / dx.doi.org/10.14201/teoredu2015272203220

MacIntyre, Alasdair. (2009). The very idea of a university: Aristotle, Newman and us. British Journal of Educational Studies, 57 (4), 347-362, doi: 10.1111/j.14678527.2009.00443.x

Marginson, Simon. (2016). Higher education and the common good. Melbourne: Melbourne University Publishing.

Maassen, Peter y Stensaker, Bjørn. (2011). The knowledge triangle, European higher education policy logics and policy implications. Higher Education, 61 (6), 757-769.

Michavila, Francisco. (2011). Principios para una educación universitaria diferente. En Fundación Europea Sociedad y Educación. Profesores y estudiantes en 
el centro de la Universidad (pp. 35-56). Madrid: Studia xxI/Fundación Europea Sociedad y Educación.

Morín, Edgar. (2010). Anotaciones para un nuevo Emilio: transmisión sistémica del conocimiento. Signo y Pensamiento, 56, 42-49.

Negrín, Olegario y Vergara, Javier. (2014). La educación en la Edad Media occidental: un tránsito a la modernización. En O. Negrín y J. Vergara (eds.). Historia de la Educación (pp. 107-152). Madrid: Dykinson.

Newman, John Henry. (1996 [1900]). Discursos sobre el fin y la naturaleza de la educación universitaria. Pamplona: Eunsa.

Nussbaum, Martha. (1997). Cultivating Humanity. A classical defense of reform in liberal education. Cambridge: Cambridge University Press.

Paleari, Stefano, Donina, Davide y Meoli, Michele. (2015). The role of the university in twenty-first century European society. The Journal of Technology Transfer, 40 (3), 369-379.

Parsons, Talcott y Platt, George M. (1973). The American University. Cambridge: Harvard University Press.

Pétriz, Felipe y Rubiralta, Màrius. (2017). Las misiones de la universidad. Cuadernos de Pedagogía, 476, 46- 50.

Piedras Monroy, Pedro Andrés. (2008). Una lectura de Humboldt: Max Weber y la universidad alemana. Arbor: Ciencia, Pensamiento y Cultura, 731, 481-493.

Pusser, Brian., Kempner, Ken., Marginson, Simon. y Ordorika, Imanol. (Eds.). (2012). Universities and the public sphere: Knowledge creation and state building in the era of globalization. New York: Routledge.

Readings, Bill. (1999). The university in ruins. Cambridge, MA: Harvard University Press.

Rhodes, Frank H. T. (2001). The creation of the future. The role of the American university. New York: Cornell University Press.

Rüegg, Walter. (1994). Prefacio. La universidad como institución europea. En W. Rüegg (ed.). Historia de la Universidad en Europa, vol. 1 (pp. 19-31). Bilbao: Universidad del País Vasco.

Rüegg, Walter. (2004). A history of the university in Europe: Universities in the Nineteenth and Early Twentieth Centuries (1800-1945). Cambridge: Cambridge University Press.

Ruiz Berrio, Julio. (1986). Algunas reflexiones sobre la historia de las universidades. Historia de la Educación: Revista Interuniversitaria, 5, 7-22.

Ruiz-Corbella, Marta. (2006). La universidad y el mercado del aprendizaje claves para comprender el concepto de competencia. En M.A. Murga y P. Quicios (coords). La reforma de la Universidad. Cambios exigidos por la nueva Europa (pp. 93-114). Madrid: Dykinson.

Ruiz-Corbella, Marta. (2016). La universidad ante los nuevos escenarios virtuales de aprendizaje. En M. A. Santos Rego (Ed.) Sociedad del Conocimiento. Aprendizaje e Innovación en la Universidad (pp. 91-114). Madrid: Biblioteca Nueva.

Ruiz-Corbella, Marta y Bautista-Cerro, María José. (2016). La responsabilidad social en la universidad española. Teoría de la Educación. Revista Interuniversitaria, 28 (1), 159-188, doi: http:/ / dx.doi.org/10.14201/teoredu2016281159188 
Scott, John. C. (2006). The mission of the university: medieval to postmodern transformations. The Journal of Higher Education, 77, 1-39.

Scott, Peter., Gallacher, Jim. y Parry, Gareth. (Eds.). (2016). New Languages and Landscapes of Higher Education. Oxford, UK: Oxford University Press.

Snow, Charles Percy. (1987). Las dos culturas y un segundo enfoque. Madrid: Alianza.

Teichler, Ulrich. (2015). Changing Perspectives: The Professional Relevance of Higher Education on the Way Towards the Highly-Educated Society. European Journal of Education, 50 (4), 461-477.

Trow, Martin. (2006). Reflections on the transition from elite to mass to universal access: Forms and phases of higher education in modern societies since WW.II. En J. J. F. Forest y P.G. Altbach (eds.). International Handbook of Higher Education (pp. 243-280). Dordrecht: Springer.

UNESCO (1998). Declaración Mundial sobre la Educación Superior en el Siglo xxi: visión y acción. Marco de acción prioritaria para el cambio y el desarrollo de la educación superior. París: unEsCo. [en línea]. [Consultado el 26/04/2017] Disponible en http://www.unesco.org/education/educprog/wche/declaration_spa.htm.

Villa, Borja. (2005). Sobre el lugar común: la Universidad humboldtiana puede ser correcta en teoría, pero no vale para la práctica: una breve introducción a tres textos de Humboldt sobre la Universidad. Logos: Anales del Seminario de Metafísica, 38, 273-281.

Wallerstein, Inmanuel. (2005). Análisis de sistemas-mundo, una introducción. México: Siglo XXI Editores.

Whitehead, Alfred N. $(1929,1951)$. The rhythm of education. En A. N. Whitehead (ed.). The aims of education (pp. 27-40). London: MacMillan.

Wittrock, Björn. (1991). ¿Dinosaurios o delfines? Origen y desarrollo de la universidad orientada hacia la investigación. Revista de Educación, 296, 73-97.

Zgaga, Pavel. (2014). The role of higher education centres in research and policy: a case from a European periphery. Studies in Higher Education, 39 (8), 1393 1404 . 
